\title{
VISUAL TOUR BASED ON PANAROMIC IMAGES FOR INDOOR PLACES IN CAMPUS
}

\author{
T. Bakirman ${ }^{\mathrm{a}}$ \\ ${ }^{a}$ YTU, Civil Engineering Faculty, 34220 Esenler Istanbul, Turkey - bakirman@yildiz.edu.tr
}

Youth Forum

KEY WORDS: Panoramic, Stitch, Visual Tour, Walkthrough

\begin{abstract}
:
In this paper, it is aimed to create a visual tour based on panoramic images for Civil Engineering Faculty in Yildiz Technical University. For this purpose, panoramic images should be obtained. Thus, photos taken with a tripod to have the same angle of view in every photo and panoramic images were created with stitching photos. Two different cameras with different focal length were used. With the panoramic images, visual tour with navigation tools created.
\end{abstract}

\section{INTRODUCTION}

Universities are complex locations for new students and visitors. Geographic Information Systems (GIS) offer solutions for people who are trying find the place which they look for and how to get there. Yildiz Technical University Davutpasa Campus is a new developing campus. Thus, a GIS has been developed for a solution to some problems (Figure 1).

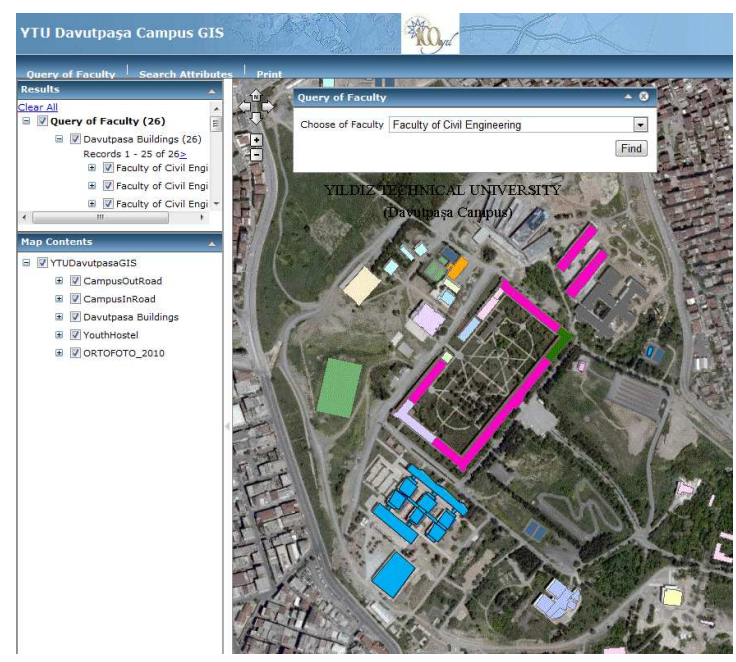

Figure 1. YTU Davutpasa Campus GIS

As computers and cameras is widely spread around the world, the use of digital images is growing. Panoramic imaging is an important part of this large use (Gledhill et al., 2003). Services based on panoramic images are becoming popular in digital map due to its expedient of virtual walking through (Chen et al., 2010).

In this paper, it is aimed to create a walkthrough for Civil Engineering Faculty based on panoramic images, which is more advanced on the visual side than GIS developed before. Panoramic visualization tools provide us a way to navigate virtual as well as real-world scenes using panoramas (Chan et al., 1999).

The panoramic images can be created with computer rendering, specialized panoramic cameras or by stitching together overlapping photographs taken with a regular camera (Chen, 1995).

In this paper, panoramic images is created with stitching method.

\section{CREATING PANORAMIC IMAGES}

Stitching images requires good set of image matching points. But, walls, doors and corridors of the building are almost identically same. Thus, it makes it harder for image matching process. Therefore, target templates are placed on appropriate places (Figure 2).

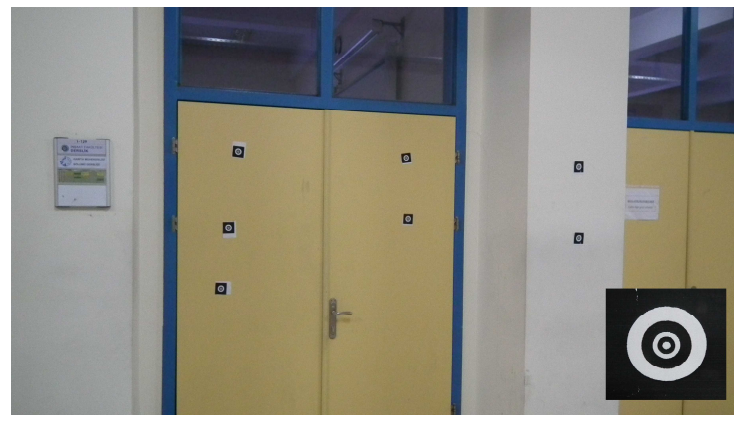

Figure 2. Target Templates

Photos were taken with tripod, so that the same angle of view can be obtained in all photos. Enough control points for a good solution are provided between sequent pair of photos (Figure 3).

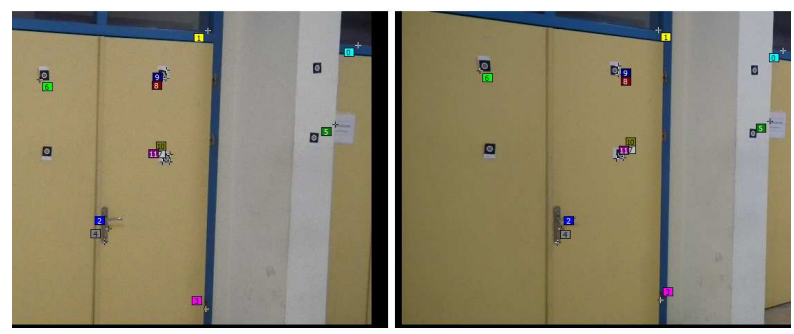

Figure 3. Control Points 
Panoramic images are created as cylindrical projection. Because, cylindrical projection doesn't have information on the top and bottom of the image (Figure 4).

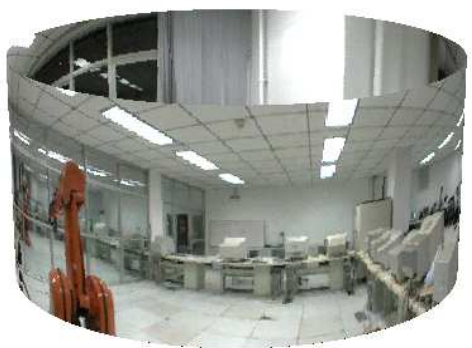

Figure 4. Cylindrical Projection (Ying et al., 2009)

Cylindrical projection allows users to have 360 degree visual in the horizontal. Also, this images can be unwrapped into a planar (Ying et al.,2009) (Figure 5).

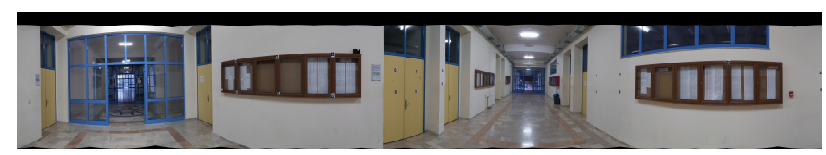

Figure 5. Unwrapped Cylindrical Panoramic Image

It was not possible to create panoramic images in the academic staff section of the faculty, because the width of the corridor is about 2.5 metres. Thus, it didn't allow a good image matching process even though so many target templates were placed (Figure 6).

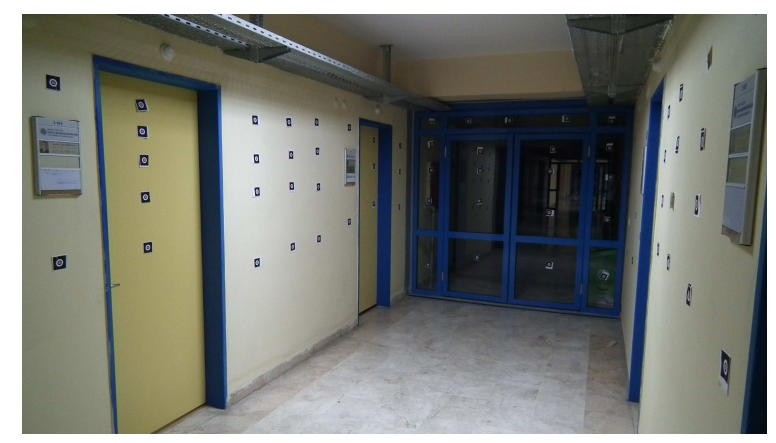

Figure 6. Academic Staff Corridor

\section{CREATING TOUR}

Panoramic images reorganized as paths. A corridor considered as a path and all panoramic in that corridor considered as a part of that path.

Every single panoramic image has different settings. According to the distance between camera and object, the field of view (fov) is set to approximately 40 degrees (Figure 7).

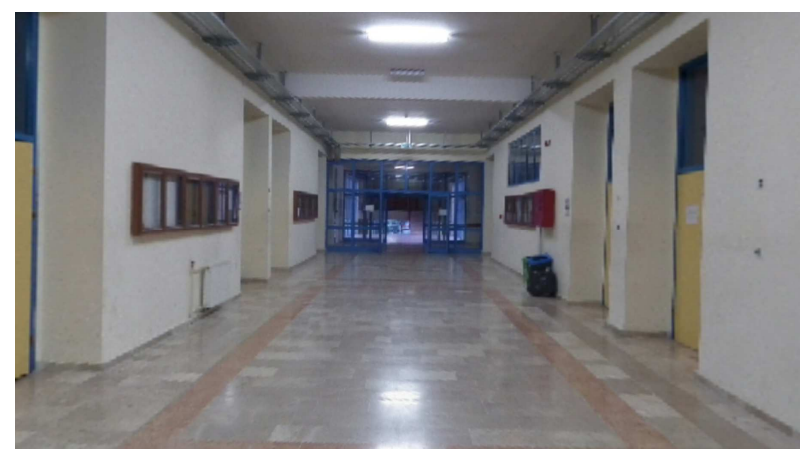

Figure 7. Panoramic Image with FOV $=40$ Degree

Most of the panoramic images contains point of interest like classroom numbers, floor names, signs etc. This points of interest determined as hotspots (Figure 8).

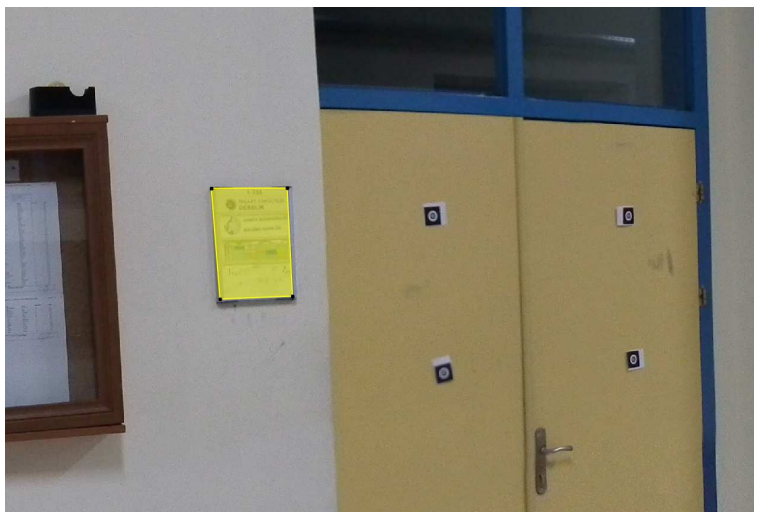

Figure 8. Adding Hotspot

These hotspots are key spots for the tour. Users should be able to get some info about the building as they virtually walk through. So hotspots are linked to a closer picture of itself, which allows to a better navigation in the tour (Figure 9).

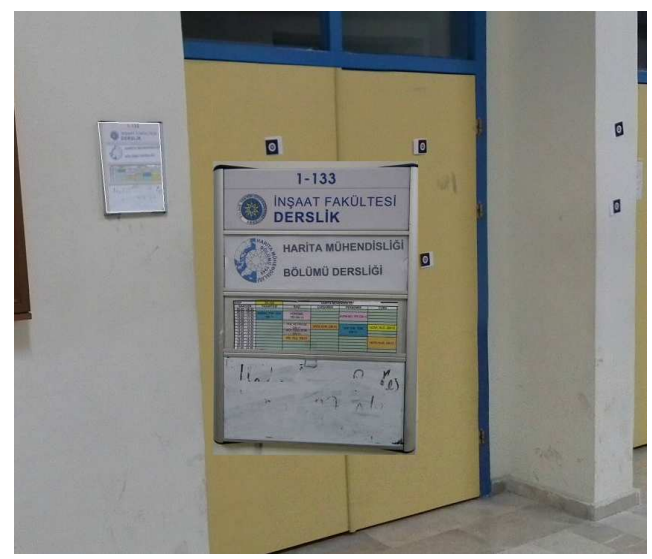

Figure 9. Hotspot Link

It is aimed that this tour to be user friendly. Thus, navigation bars and list panoramic images added to the tour which allows user to navigate between panoramic images easily (Figure 10). 


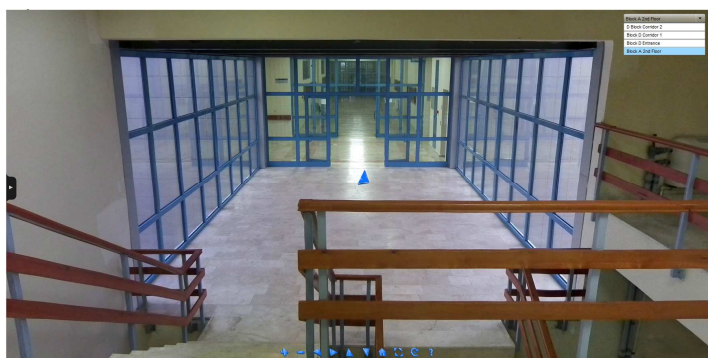

Figure 10. Navigation Tools

\section{CONCLUSION AND FUTURE}

Visual tour with panoramic images is created for Civil Engineering Faculty of Yildiz Technical University. The tour is build as html and swf format, which can be easily navigated from internet.

For the future works, other tours for all the faculties in the campus can be created and with combining these tours with street view of the campus, virtual reality of the whole campus can be obtained.

\section{REFERENCES}

Chan, Y. et al., 1999. A Panoramic-based Walkthrough System using Real Photos. Computer Graphics and Applications, pp. $231-240$.

Chen, M. et al., 2010. Automatic Annotation Of GeoInformation In Panoramic Street View By Image Retrieval. In : International Conference on Image Processing. Hong Kong, Vol. XVII, pp. $2381-2384$.

Chen, S. E., 1995. An Image Based Approach To Virtual Environment Navigation. In : SIGGRAPH'95. Los Angeles, pp. $29-38$.

Glendhill, D. et al., 2003. Panoramic Imaging - A Review. Computers \& Graphics, 27, pp. $435-445$.

Ying., X. et al., 2009. Walkthrough in Large Environments Using Concatenated Panoramas. In : IEEE International Conference on Robotics and Biomimetics, Guilin, China, pp. $286-291$. 